\title{
Hepatitis C, an unusual cause of inflammatory arthritis: a case report
}

\author{
Authors: Fiona Coath and Poonam Sharma
}

\section{Case}

A 51-year-old gentleman of Pakistani origin, living in the UK for the last 20 years, presented to the rheumatology department with a 10-year history of widespread joint pain and deformity. He had a background of idiopathic thrombocytopaenic purpura (ITP), chronic folliculitis, osteoporosis and hepatitis C virus (HCV) in 2008. He was unfortunately lost to follow up after the initial HCV diagnosis and not fully treated. On examination he had fixed flexion deformity of the right elbow with fusion of the right wrist but no palpable synovitis.

Up-to-date serology confirmed positivity for HCV antibodies, genotype 3, with a low viral load. Liver transaminases were at baseline following significant elevation during the acute infective period. There was persistent thrombocytopaenia, but in the context of a normal liver fibroscan and spleen size, felt to be secondary to ITP rather than splenic sequestration. Liver synthetic function was otherwise normal. Polyclonal gamma-globulinaemia would be explained by chronic viral infection in this case. From an arthritis perspective autoimmune serology was unremarkable (negative rheumatoid factor (RF), anti-cyclic citrullinated peptide antibodies (ACCPA), cryoglobulins, atypical anti-neutrophil cytoplasmic antibodies but myeloperoxidase and proteinase 3 negative). Imaging was consistent with HCV-associated arthritis, with plain film demonstrating wrist fusion but no erosions. Magnetic resonance imaging of the right hand demonstrated fused carpals and ankylosis at the 2 nd and 3rd carpometacarpal joints, but no active synovitis.

The diagnosis was HCV-related arthropathy (HCVrA). Treatment for this patient required addressing the underlying $\mathrm{HCV}$, as well as symptomatic management of his arthritis. In the absence of erosions, active synovitis or other features to suggest concomitant rheumatoid arthritis, there was no indication to start disease modifying antirheumatic drugs at present.

\section{Discussion}

Extra-hepatic complications of HCV are common and estimated to affect $74 \%$ of patients, with approximately $4 \%$ suffering from HCVrA. ${ }^{1,2}$ A larger proportion will experience arthralgia rather than true arthritis. Development of arthritis does not appear to correlate with the type or severity of HCV, so should be considered in all patients complaining of joint pain. ${ }^{1}$ Diagnosis can be challenging, as presentation is often polyarticular and symmetrical, mimicking a rheumatoid arthritis (RA) pattern. The distinction is important, as management differs. Serology can be unhelpful, with positive RF seen in $40-70 \%$ of $\mathrm{HCVrA}{ }^{3}$ ACCPA is more specific for RA, so if positive one would need to consider treating for RA in addition to HCV. HCVrA is associated with a lack of erosions, although in early RA this may also be the case. However, better outcomes are seen in early treated RA, so emphasis should be placed on adequate screening and prompt referral to rheumatology.

\section{Learning points}

> Effective management of HCVrA requires a multidisciplinary approach between rheumatology and hepatology.

> Patients with a new diagnosis of HCV should be screened for the presence of musculoskeletal symptoms and if there are concerns, referred for specialist assessment as soon as possible.

> In patients presenting with polyathropathy in the context of normal autoimmune serology, clinicians should consider screening for secondary infective causes such as hepatitis.

\section{Conflict of interest statement}

None declared.

\section{References}

1 Kemmer NM, Sherman KE. Hepatits C-related arthropathy: Diagnostic and treatment considerations. J Musculoskelet Med 2010;27:351-4.

2 Siloşi L, Boldeanu L, Biciuşca $V$ et al. Serum biomarkers for discrimination between hepatitis $\mathrm{C}$-related arthropathy and early rheumatoid arthritis. Int J Mol Sci 2017;18:1304.

3 Ferucci ED, Choromanski TL, Varney DT et al. Prevalence and correlates of hepatitis $C$ virus-associated inflammatory arthritis in a population-based cohort. Semin Arthritis Rheum 2017;47:445-50. 